\title{
Uterine Obstruction, CTCAE
}

National Cancer Institute

\section{Source}

National Cancer Institute. Uterine Obstruction, CT CAE. NCI Thesaurus. Code C143918.

A disorder characterized by blockage of the uterine outlet. 